\title{
Bone material strength index as measured by impact microindentation is altered in patients with acromegaly
}

\author{
F Malgo', N A T Hamdy'1, T J Rabelink², H M Kroon 33, K M J A Claessen', \\ A M Pereira', N R Biermasz ${ }^{4}$ and N M Appelman-Dijkstra ${ }^{1}$
}

Centre for Bone Quality, 'Division Endocrinology and, '2Division Nephrology, ${ }^{3}$ Department of Radiology, and ${ }^{4}$ Centre for Endocrine Tumours Leiden, Department of Medicine, Division Endocrinology,

Leiden University Medical Center, Leiden, The Netherlands
Correspondence should be addressed to N M Appelman-Dijkstra Email

N.M.Appelman-Dijkstra@ lumc.nl

\begin{abstract}
Objective: Acromegaly is a rare disease caused by excess growth hormone (GH) production by the pituitary adenoma. The skeletal complications of GH and IGF-1 excess include increased bone turnover, increased cortical bone mass and deteriorated microarchitecture of trabecular bone, associated with a high risk of vertebral fractures in the presence of relatively normal bone mineral density (BMD). We aimed to evaluate tissue-level properties of bone using impact microindentation (IMI) in well-controlled patients with acromegaly aged $\geq 18$ years compared to 44 controls from the outpatient clinic of the Centre for Bone Quality.

Design and methods: In this cross-sectional study, bone material strength index (BMSi) was measured in 48 acromegaly patients and 44 controls with impact microindentation using the osteoprobe.

Results: Mean age of acromegaly patients (54\% male) was 60.2 years (range 37.9-76.5), and 60.5 years (range 39.8-78.6) in controls ( $50 \%$ male). Patients with acromegaly and control patients had comparable BMI $\left(28.2 \mathrm{~kg} / \mathrm{m}^{2} \pm 4.7 \mathrm{vs} 26.6 \mathrm{~kg} /\right.$ $\left.\mathrm{m}^{2} \pm 4.3, P=0.087\right)$ and comparable BMD at the lumbar spine $\left(1.04 \mathrm{~g} / \mathrm{cm}^{2} \pm 0.21 \mathrm{vs} 1.03 \mathrm{~g} / \mathrm{cm}^{2} \pm 0.13, P=0.850\right)$ and at the femoral neck $\left(0.84 \mathrm{~g} / \mathrm{cm}^{2} \pm 0.16\right.$ vs $\left.0.80 \mathrm{~g} / \mathrm{cm}^{2} \pm 0.09, P=0.246\right)$. BMSi was significantly lower in acromegaly patients than that in controls $(79.4 \pm 0.7$ vs $83.2 \pm 0.7 ; P<0.001)$.

Conclusion: Our data indicates that tissue-level properties of cortical bone are significantly altered in patients with controlled acromegaly after reversal of long-term exposure to pathologically high GH and IGF-1 levels. Our findings also suggest that methods other than DXA should be considered to evaluate bone fragility in patients with acromegaly.
\end{abstract}

\section{Introduction}

Acromegaly is a rare endocrine disease characterized by excess circulating growth hormone $(\mathrm{GH})$ and insulinlike growth factor-1 (IGF-1) levels, usually caused by an adenoma of the anterior pituitary gland. GH excess results in morbidity of multiple organ systems, including generalized tissue hypertrophy, cardio-metabolic disorders (diabetes mellitus and hypertension and cardiomyopathy) and arthropathy. Acromegaly has been shown to be
두 2017 European Society of Endocrinology Printed in Great Britain associated with an increased risk of vertebral fractures in the presence of a relatively normal bone mineral density (BMD), and it has also been shown that these fractures may progress despite adequate control of disease activity $(1,2,3,4,5)$. Patients with active acromegaly demonstrate high bone turnover associated with increased cortical BMD in the presence of stable trabecular bone mass. Iliac crest biopsies indeed show an increase in bone 
remodeling compared to that in healthy controls (6). Trabecular connections are lost, due to the high bone turnover, resulting in altered bone microarchitecture, which also persists after successful induction of remission and restoration of bone remodeling rates $(7,8)$. In a study with HRpQCT, structural measurements confirmed this altered bone microarchitecture in patients with active as well as controlled acromegaly. This included deteriorated trabecular microarchitectural parameters in the distal radius and distal tibia and increased cortical volumetric BMD in the distal tibia in patients with active disease (9). Areal BMD is often reported to be normal in these patients, although some studies do suggest an association with low bone mass $(2,10)$. Overall, current methods do not provide an adequate explanation for the increased fracture risk in these patients. However, in a recently published study evaluating the association between bone microstructure, as measured by high-resolution cone beam computed tomography, and vertebral fractures, the authors found deteriorated microstructure in both the trabecular and the cortical bone compartments of acromegaly patients with vertebral fractures compared to those without vertebral fractures (11).

There are no published data available on the assessment of material properties of cortical bone in patients with acromegaly; however, there are published data on material properties of trabecular bone in patients with acromegaly (12). The technique of impact microindentation (IMI) was introduced as a reference point indentation method to acquire Bone Material Strength index measurements in vivo and is used as a surrogate to assess tissue-level properties of bone. Previous studies have shown deteriorated material properties in postmenopausal women with osteoporotic fractures, atypical femoral fractures, with type 2 diabetes mellitus and in patients with fragility fractures $(13,14,15,16)$. Although IMI reflects cortical bone properties rather than trabecular bone properties, there is evidence that the elevated GH levels seen in acromegaly affect both compartments $(6,11)$. As current diagnostic tools do not adequately identify acromegaly patients who are at high risk for vertebral fractures, new methods are needed.

In this study, we aimed to evaluate previously reported findings of poor bone material properties in patients with acromegaly by evaluating whether BMSi is different between patients with acromegaly in remission and controls without acromegaly. In addition, we also aimed to evaluate whether BMSi was different in patients with acromegaly, with or without vertebral fractures.

\section{Patients and methods}

\section{Study design}

We performed a cross-sectional study to compare Bone Material Strength index (BMSi) between patients with well-controlled acromegaly and non-acromegaly control patients with osteopenia or normal bone mineral density (BMD), with or without fractures. Patients were studied at the outpatient clinics of the Leiden University Medical Center. The Medical Ethics Committee of the Leiden University Medical Center reviewed and approved the study, and all patients gave written informed consent to participate in the study.

\section{Patients with acromegaly}

All patients aged 18 years or older with well-controlled acromegaly attending the outpatient clinic of the Department of Endocrinology of the Leiden University Medical Center were identified from hospital records. 170 patients with acromegaly, 73 of whom fulfilled the inclusion criteria and were invited to participate in the study. Forty-eight patients responded positively and were included in the study (Fig. 1). Of these patients, 26 (54\%) had been evaluated in previously reported studies from our center $(2,4)$.

In this study, well-controlled acromegaly was defined as serum IGF-1 levels within the normal age range after surgery, radiotherapy, medical treatment or a combination thereof, and when required, additional confirmation of remission from glucose-suppression tests. Oral glucose tolerance tests were performed yearly to assess disease activity, except in patients receiving medical treatment (17).

\section{Controls}

Controls were recruited from the outpatient clinic of the Centre for Bone Quality or from the regional Fracture Liaison Service of the Leiden University Medical Center and were matched on age and BMD. A number of these control patients have also been reported in an earlier study ( $n=12)$ (16). Control patients who reported fragility fractures or who had grade 2 or 3 morphometric vertebral fractures were excluded from the study. A fragility fracture was defined as any low-energy fracture, excluding those of the hands, feet and skull. Control patients with fractures after a high-energy trauma or grade 1 morphometric vertebral fractures were included in the study. 
9 referred to other center

4 not able to obtain consent

13 recorded wish not to be involved in studies

36 acromegaly not in complete remission

13 using bone modifying drugs

7 inability to measure femoral neck BMD

14 comorbidity

1 metabolic bone disease (fibrous dysplasia)

25 refised to partipare

73 patients invited to

participate in study

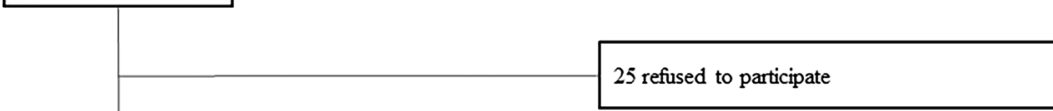

BMSi measured

in 48 patients

Both acromegaly and non-acromegaly control patients were excluded if they had a metabolic bone disease other than osteoporosis; any untreated endocrine disorder; severe liver insufficiency or chronic kidney disease stage IV or V; have had bilateral hip replacement; were currently using aromatase inhibitors or androgen deprivation therapy; and were currently using or had used bisphosphonates, denosumab, selective estrogen receptor modulators (SERMs), strontium ranelate or recombinant PTH. Furthermore, patients were excluded if they had a localized infection of the tibia or were unable to provide informed consent. Current or past use of glucocorticoids was also an exclusion criterion with the exception of hydrocortisone supplementation for secondary adrenal insufficiency.

\section{Methods}

Medical history data including date of start of treatment, type of treatment (surgery, radiotherapy, medication use or combined), date of normalization of IGF-1, data on other pituitary function tests, menopausal status, previous or current medication use and fracture history were collected at the time of inclusion in the study. Daily calcium intake was calculated and clinical risk factors for fracture as used in the FRAX algorithm were obtained from each patient.

\section{Figure 1}

Flowchart of patient recruitment.

\section{Laboratory investigations}

Serum calcium, phosphate, albumin and creatinine were measured using semi-automated techniques. Plasma intact PTH was measured by the IMMULITE 2500 (Siemens Diagnostics), and serum 25-hydroxyvitamin D was measured using the 25-OH-vitamin D TOTAL assay (DiaSorin D.A./N.V.). Procollagen type 1 amino-terminal propeptide (P1NP) and $\beta$-crosslaps were measured by an electrochemoluminescent immunoassay with a Modular Analytics E-170 system (Roche Diagnostics).

Serum IGF-1 levels (nmol/L) were measured using an immunometric technique on an IMMULITE 2500 system (Diagnostic Products Corporation, Deerfield, IL, USA). The intra-assay variations at mean plasma levels of 8 and $75 \mathrm{nmol} / \mathrm{L}$ were $5.0 \%$ and $7.5 \%$ respectively. IGF-1 levels were expressed as SDS, using $\lambda-\mu-\sigma$ smoothed reference curves based on measurements in 906 healthy individuals $(18,19)$.

Serum GH levels were measured with a immunofluorometric assay (Siemens Diagnostics) calibrated against World Health Organization (WHO) National Institute for Biological Standards and Control (NIBSC) 2nd International Standard 98/574. Values were multiplied by 1.02 .

\section{Bone mineral density}

BMD was measured at the lumbar vertebrae (L1-L4) and at the left and right femoral neck using dual-energy 
X-ray absorptiometry (DXA) (Hologic QDR Discovery A (Hologic, Bedford, MA, USA)) at the time of indentation. NHANES III reference values compatible with reference values of the Dutch population were used to calculate T-scores. Normal bone mineral density, osteopenia or osteoporosis was diagnosed using WHO criteria.

\section{Spinal radiographs}

Conventional antero-posterior and lateral radiographs of the thoracic and lumbar spine were performed following standard protocols, at a focus-detector distance of $115 \mathrm{~cm}$, with the detector centralized on Th7 for the thoracic spine and on L3 for the lumbar spine at the time of inclusion. The semi-quantitative method of Genant was used to assess the presence and grading of vertebral fractures. Vertebral fractures were scored as grade 1 (reduction in anterior, middle and/or posterior height between $20 \%$ and $25 \%$ ), grade 2 (reduction in anterior, middle and/ or posterior height between $25 \%$ and $40 \%$ ) or grade 3 (reduction $>40 \%$ in anterior, middle and/or posterior height) (20), without a history of high-energy trauma.

Radiographs were independently assessed by two of the authors ( $\mathrm{N} \mathrm{A-D}$ and $\mathrm{H} \mathrm{K}$ ). Consensus was reached in case of difference in assessment.

\section{Impact microindentation}

A reference point indentation tool, the Osteoprobe, specifically designed for in vivo measurements of Bone Material Strength index (BMSi) in humans and large animals (21), was used as a surrogate to evaluate bone material properties (16). Impact microindentation is performed on the midshaft of the tibia by inserting a test probe in the skin until the bone surface is reached. By indenting the bone surface, the resistance of the bone tissue to fracture is evaluated (Fig. 2). The first studies performed in humans with a previous version of the technique have shown that postmenopausal women with osteoporotic fracture and with atypical femoral fracture have significantly higher indentation distance increase (IDI) than controls without fractures. Further studies using the impact microindentation technique demonstrated that patients with suspected deteriorated material properties such as patients with fragility fractures or patients with type 2 diabetes mellitus had significantly lower BMSi values $(13,14,15,16)$. The measurement site was defined as the mean distance between the distal apex of the patella and medial malleolus. Local anesthesia (Lidocaine 1\%) was applied at the measurement site.

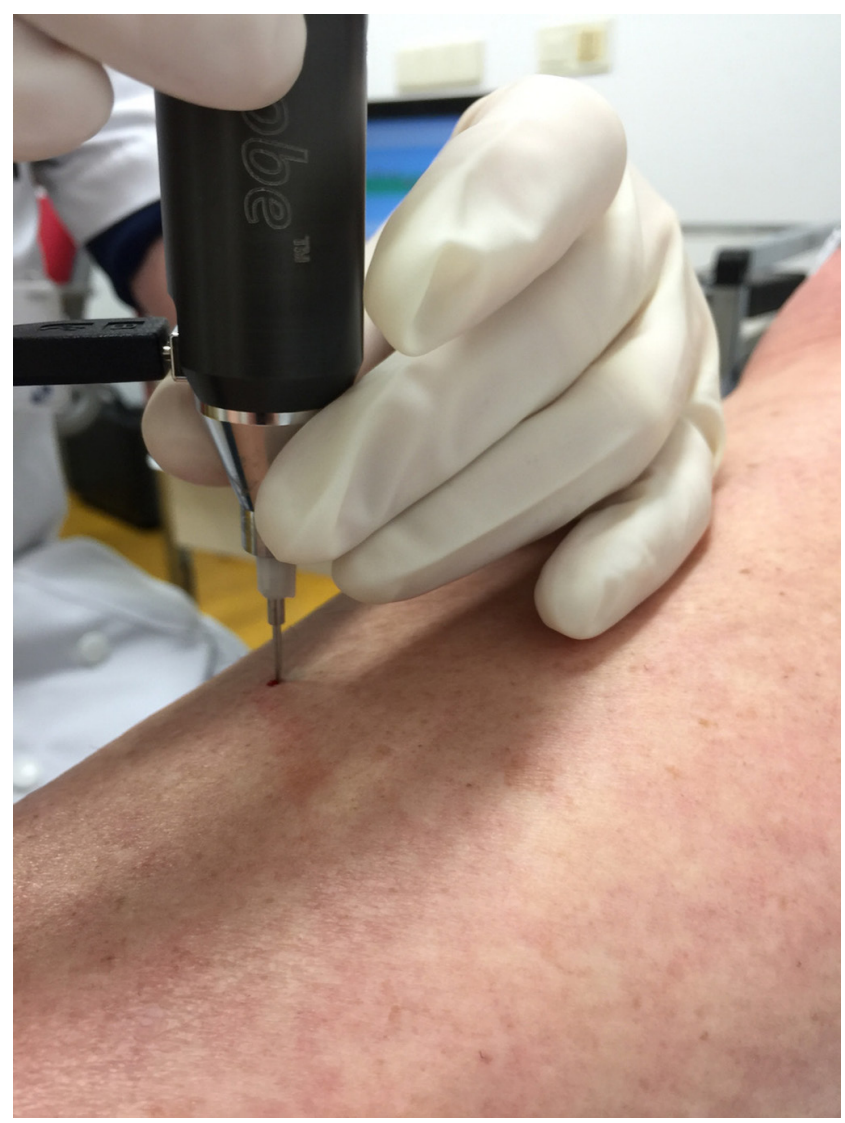

\section{Figure 2}

Positioning of the test probe perpendicular to the bone surface.

The test probe was inserted at the site of interest after successful anesthesia of the skin and periosteum and was pushed gently until it reached the bone surface. It was ensured that the test probe was always perpendicular to the bone surface during measurements. The operator was not allowed to check the measurements on the computer screen before these were classified as 'well performed', 'adequate' or 'poorly performed'. Measurements were classified as 'poorly performed' if the measurement probe slipped or if the subject moved his/her leg. At least five adequate measurements were obtained from each patient, followed by five additional measurements performed on a polymethylmethacrylate (PMMA) calibration phantom. The number of measurements ranged from 10 to 24 . The resultant outcome BMSi was defined as 100 times the ratio of the harmonic mean indentation distance increase from impact into the PMMA calibration phantom divided by the indentation distance increase from impact into bone (21).

The intra-observer coefficient of variation $(\mathrm{CV})$ was $2.2 \%$. Ten subjects were measured twice on the same 
leg. The distance between the two measurement sites was $2 \mathrm{~cm}$.

\section{Statistical analysis}

Analyses were performed using SPSS software for Windows (version 23.0; SPSS Inc.). Data are expressed as mean \pm S.D. unless otherwise stated. Normality assumptions were checked by the evaluation of normality plots and by inspection of histograms of residuals from miscellaneous regression models. Differences between groups were assessed using a two-sample $t$ tests or chi-square tests. Pearson/Spearman correlation coefficients were used to express correlations between IGF-1 levels, duration of disease remission and BMSi values. Analysis of variance models with BMSi as outcome variable adjusted for age was used to compare BMSi values between patients and controls. Differences were considered to be significant at $P<0.05$.

\section{Results}

We included 48 patients with acromegaly and 44 controls who were comparable in gender, age, BMI and BMD at the lumbar spine and at the femoral neck (Table 1). The two groups were also comparable regarding the number of patients who had sustained a non-vertebral fracture, 11
(23\%) in acromegaly patients and 11 (25\%) in controls. There were more acromegaly patients with a vertebral deformity than controls (28 (58\%) vs 7 (16\%); $P<0.001)$.

In the acromegaly group, 27 patients (56\%) were in remission after surgery, 7 of whom received additional radiotherapy. 21 (44\%) patients were controlled with ongoing medical treatment with somatostatin analogs only $(n=14)$, Pegvisomant, a GH receptor antagonist, only $(n=3)$ or combination therapy $(n=4), 1$ of whom had received radiotherapy, 16 of whom had surgery and 4 of whom had both. The mean duration of acromegaly remission was 16.1 years (range $0.5-37.8$ years). There were 8 patients with type 2 diabetes mellitus, 3 of whom were conservatively treated and 5 received drug therapy (4 oral anti-diabetic agents and 1 insulin therapy), 11 with treated hypothyroidism, 10 with treated adrenal insufficiency and 9 with treated hypogonadism.

\section{Bone Material Strength index}

Patients with acromegaly had significantly lower BMSi values compared to controls $(79.4 \pm 0.7$ vs $83.2 \pm 0.7$; $P<0.001)$, also after adjustment for age $(79.4 \pm 0.7$ vs $83.2 \pm 0.7 ; P<0.001$ ) (Fig. 3). This finding remained if patients with type 2 diabetes mellitus were excluded $(79.2 \pm 0.8$ vs $83.2 \pm 0.7 ; P<0.001)$. Although numbers

Table 1 Characteristics of patients with acromegaly and non-acromegaly controls. Values are expressed as mean \pm S.D.

\begin{tabular}{l}
\hline \\
\hline Age (years) \\
Male/female \\
BMI $\left(\mathrm{kg} / \mathrm{m}^{2}\right)$ \\
Fracture \\
Vertebral fracture \\
Grade 1 \\
Grade $2 / 3$ \\
Smoking $(\%)$ \\
Alcohol use $>3 \mathrm{IU} /$ day $(\%)$ \\
Glucocorticoids $(\%)$ \\
PTH $(\mathrm{pmol} / \mathrm{L})$ \\
Calcium $(\mathrm{mmol} / \mathrm{L})$ \\
25-OH D (nmol/L) \\
Creatinine $(\mu \mathrm{mol} / \mathrm{L})$ \\
GH (mU/L) \\
IGF-1 $(\mathrm{nmol} / \mathrm{L})$ \\
IGF-1 s.D. \\
LS BMD $\left(\mathrm{g} / \mathrm{cm}^{2}\right)$ \\
T-score $\mathrm{LS}$ \\
FN BMD $\left(\mathrm{g} / \mathrm{cm}^{2}\right)$ \\
T-score FN
\end{tabular}

\begin{tabular}{c}
\hline Acromegaly $(n=48)$ \\
$60.2 \pm 11.0$ \\
$26 / 22$ \\
$28.2 \pm 4.7$ \\
$11(23 \%)$ \\
$28(58 \%)$ \\
$28(58 \%)$ \\
$11(23 \%)$ \\
$6(13 \%)$ \\
$5(10 \%)$ \\
$8(17 \%)$ \\
$4.6 \pm 2.8$ \\
$2.38 \pm 0.11$ \\
$67.3 \pm 28.5$ \\
$78.8 \pm 14.3$ \\
$4.0 \pm 5.5$ \\
$19.5 \pm 7.6$ \\
$1.0 \pm 1.3$ \\
$1.04 \pm 0.21$ \\
$-0.3 \pm 1.8$ \\
$0.84 \pm 0.16$ \\
$-0.6 \pm 1.2$
\end{tabular}

\begin{tabular}{c}
\hline Control $(n=44)$ \\
$60.5 \pm 8.5$ \\
$22 / 22$ \\
$26.6 \pm 4.3$ \\
$11(25 \%)$ \\
$7(16 \%)$ \\
$7(16 \%)$ \\
0 \\
$8(18 \%)$ \\
$8(18 \%)$ \\
$5(11 \%)$ \\
$3.3 \pm 1.5$ \\
$2.39 \pm 0.10$ \\
$67.3 \pm 27.6$ \\
$76.1 \pm 18.4$ \\
- \\
- \\
- \\
$1.03 \pm 0.13$ \\
$-0.3 \pm 1.2$ \\
$0.80 \pm 0.09$ \\
$-0.8 \pm 0.8$
\end{tabular}

\begin{tabular}{c}
\hline P-value \\
\hline 0.849 \\
0.689 \\
0.087 \\
0.815 \\
$<0.001$ \\
$<0.001$ \\
0.001 \\
0.449 \\
0.304 \\
0.466 \\
0.014 \\
0.473 \\
0.944 \\
0.471 \\
\\
\end{tabular}

BMI, body mass index; IU, international unit; PTH, parathyroid hormone; GH, growth hormone; IGF-1, insulin-like growth factor 1; LS, lumbar spine; FN, femoral neck; BMD, bone mineral density. Reference range: PTH (0.7-8.0 pmol/L); Calcium ( $2.15-2.55 \mathrm{mmol} / \mathrm{L}) ; 25-\mathrm{OH} \mathrm{D}(50-250 \mathrm{nmol} / \mathrm{L})$; Creatinine $(64-104 \mu \mathrm{mol} / \mathrm{L}$ for $\mathrm{male}, 49-90 \mu \mathrm{mol} / \mathrm{L}$ for female); GH (0.00-7.25 mU/L); IGF-1 (6.8-26.5 nmol/L). 
A

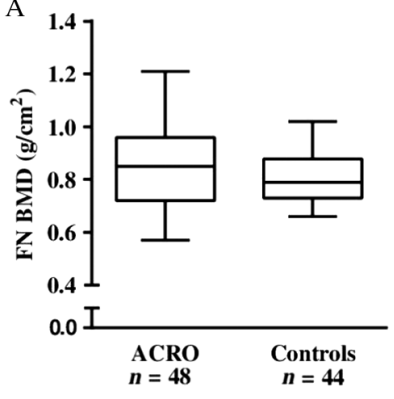

B

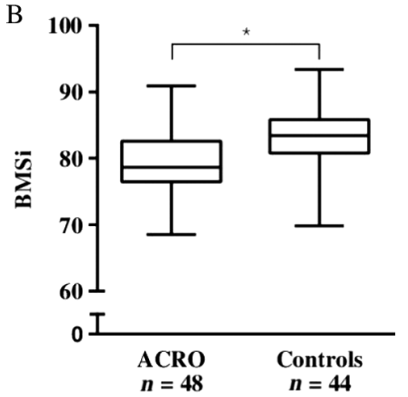

Figure 3

(A) Mean femoral neck Bone Mineral Density (FN BMD) and (B) Bone Material Strength index (BMSi) in patients with acromegaly and non-acromegaly controls. Data are shown in box-whisker plots and statistical differences are displayed for BMSi. Boxes indicate median and inter-quantile range. Bars indicate minimum and maximum values.

are small, there was no statistically significant difference in BMSi in patients with acromegaly regardless of the therapeutic intervention used to normalize IGF-1 levels (surgery: $81.1 \pm 1.0$, surgery and radiotherapy: $77.9 \pm 1.7$, medical treatment: $78.4 \pm 1.0 ; P=0.114$ ).

\section{Factors affecting BMSi in acromegaly patients}

In the group of patients with acromegaly, there was a significant relationship between BMSi and age ( $r=0.291, P=0.045)$. BMSi was not correlated with other clinical characteristics including BMI and duration of remission. There was also no relationship between BMSi and any biochemical parameter measured including parameters of calcium metabolism, serum levels of GH and IGF-1, gonadal hormone status or with BMD. There was no difference in BMSi values between male or female patients $(79.5 \pm 0.9$ vs $79.2 \pm 1.0 ; P=0.804)$ or between patients with or without a non-vertebral fracture $(80.2 \pm 1.4$ vs $79.1 \pm 0.8 ; \quad P=0.511)$. There was no statistically significant difference in BMSi between patients with $(n=28)$ or without a vertebral fracture $(n=20)$; Supplementary Fig. 1 (see section on supplementary data given at the end of this article), also in the comparison between patients with Genant $\geq$ grade 2 vertebral fractures $(n=11)$ and patients with Genant $\leq$ grade 1 vertebral fractures $(n=37)(80.3 \pm 1.4$ vs $79.1 \pm 0.8 ; P=0.466)$.

In the control group, there was a significant inverse relationship between BMSi and age $(r=-0.457, P=0.002)$, but no relationship between BMSi and any other parameter measured, including BMD.

\section{Discussion}

To our knowledge, this is the first study examining tissuelevel properties of cortical bone in patients with wellcontrolled acromegaly, previously shown to be at high risk for vertebral fractures independently of BMD. Our data show significantly lower Bone Material Strength index (BMSi) values in patients with well-controlled acromegaly than those in controls with comparable age and BMD. There was no difference in BMSi within the group of patients with acromegaly, in the presence or absence of vertebral fractures or in the presence or absence of treated hypogonadism.

The reported prevalence of vertebral fractures in patients with acromegaly is high, ranging from 53\% to $59 \%(1,2,10)$. Longitudinal data also show that vertebral fractures continue to progress in controlled disease in patients both with and without previous vertebral fractures $(4,5)$. This increased vertebral fracture risk in the presence of normal or high-normal BMD in patients with well-controlled acromegaly suggests that components other than bone mass may influence bone strength in these patients, such as bone microarchitecture or bone material properties. Indeed, histological findings from bone biopsies of the iliac crest performed in patients with active acromegaly confirm this suggestion by showing altered histomorphometric parameters of bone, particularly at cortical sites (6). During active acromegaly, bone turnover is increased, and this is associated with an increase in cortical bone mass. Trabecular bone mass remains stable although trabecular connections are lost due to the high bone turnover $(6,7,8)$. Further reported data have also shown an increase in the diameter of trabeculae, suggesting microarchitectural changes in the trabecular compartment of bone (22). These findings were further confirmed in a study using HRpQCT, which demonstrated altered microarchitecture of trabecular bone in eugonadal acromegalic patients compared to those in healthy controls (9). The observations from the same study regarding the cortical compartment appear to be in contrast with the findings from our study, as cortical volumetric BMD was increased in active acromegalic patients. However, these patients were compared with patients with controlled acromegaly, and our cohort consists of well-controlled acromegaly patients only.

A recent study in transgenic bGH mice demonstrated poor microarchitecture of both cortical and trabecular compartments of the tibia. This is an animal model with pathologically elevated GH levels occurring in utero and in adult life. Although this mouse model develops 
increased GH levels during skeletal development and is therefore not fully comparable with the manifestation of GH excess after epiphyseal closure in acromegaly, it may provide interesting observations on the effect of GH on the skeleton. The bGH mice were shown to have a significantly lower trabecular number and lower trabecular thickness than control mice, in the presence of overall larger bones and increased tibia length. bGH mice were also found to have increased cortical bone perimeter and cross-sectional area in the tibia, compared with control mice, despite lower cortical bone thickness (23). Cortical BMD was also significantly lower in the vertebrae of bGH mice compared to that in control mice. Vertebral trabecular BMD was comparable but trabecular thickness was decreased. Mechanical tests performed on the femora showed significantly lower mechanical properties, including ultimate stress and Young's modulus, in bGH mice compared to littermate controls. These results suggest that the cortical compartment in vertebrae is perhaps more affected than its trabecular compartment as a result of exposure to excess growth hormone. These data may provide some further understanding of our finding of a lower Bone Material Strength index, which mainly measures cortical bone properties, in patients with acromegaly with and without fractures.

The observation that BMSi of acromegaly patients with vertebral fractures was not statistically different from BMSi of acromegaly patients without vertebral fractures might be in keeping with previous suggestions that acromegaly affects not only the trabecular compartment but also the cortical compartment. For instance, Vallassi et al. reported that both cortical and trabecular volumetric BMD were reduced at the proximal femur in 35 acromegaly patients, regardless of gender, gonadal status and disease activity (24). Recently, Maffezzoni et al. reported in 40 patients with acromegaly that those with vertebral fractures $(n=15)$ had lower bone volume/ trabecular volume ratio, greater mean trabecular separation and higher cortical porosity compared to those without vertebral fractures $(n=25)$ (11). However, as is also seen in our study, group sizes are small due to the rarity of the disease and preclude firm conclusions.

Impact microindentation is an emerging reference point indentation technique that measures tissue-level properties of cortical bone in vivo at the midshaft of the tibia, thus reflecting tissue-level properties of cortical rather than trabecular bone. Although this technique appears as a promising alternative to assess bone fragility, data on fractures (including vertebral fractures) and Bone Material Strength index are relatively limited.
A preliminary report of postmenopausal women with (49\% vertebral fracture, 51\% non-vertebral fracture) or without fractures found lower BMSi values in fracture patients and a significant inverse relationship between BMSi and grade of vertebral fracture (25), whereas a recent study in elderly community-dwelling women showed no relationship between prevalent vertebral fractures and BMSi (26). Within our own cohort of patients with low bone mass and fragility fractures, we demonstrated that BMSi was low irrespective of the type of fracture: vertebral, nonvertebral or a combination of the two fracture types (27). The difference in BMSi between patient groups (including fracture patients) and controls in reported literature is variable from $4.5 \%$ up to $9.2 \%(15,16,25,28)$. Although our findings are in keeping with those from other groups, the predictive value remains to be determined.

This study has strengths as well as limitations. A major strength of the study is having had access to a large cohort of well-characterized patients with acromegaly, all hormonally and metabolically well controlled. A limitation of the study is the cross-sectional design. It remains to be established whether BMSi is a predictor for vertebral fractures in acromegaly patients in prospective studies. Also, we were only able to obtain areal BMD data from all patients but not volumetric BMD in any. GH significantly increases the size of the skeleton in patients with acromegaly and areal BMD does not adjust for the size of bone. Although acromegaly is associated with comorbidities such as type 2 diabetes mellitus and hypogonadism, which in itself may contribute to bone fragility, all endocrine deficiencies were adequately supplemented and diabetes was well controlled by their treating physician as required. Lastly, patients with a very high fracture risk could not be included in this study, because these patients were currently or had been previously treated with bone-modifying agents.

Findings from our study add to the accumulating evidence for the association of acromegaly with structural changes of bone, also after adequate control of growth hormone production. This is in keeping with findings from two recent studies that reported a decrease in trabecular bone score (TBS) in the presence of an increase in lumbar spine BMD after successful treatment of acromegaly (29), and a decrease in cortical and trabecular volumetric BMD measured at the femur, independently of acromegaly disease status (24).

In keeping with our previous observation, we found a strong inverse relationship between BMSi and age in our control group. In contrast, we found a positive relationship between BMSi and age in patients with 
acromegaly. A possible explanation of this contrasting finding is that older patients had longer remission periods and the skeleton may have had more time to recover from the effects of high GH levels. This may suggest that acromegaly patients with more recent exposure of high $\mathrm{GH}$ levels (the young patients with acromegaly) have lower BMSi values than patients with acromegaly with longer duration of remission. However, we did not find a relationship between BMSi and the duration of remission in our series ( $r=0.220, P=0.133)$. There was no difference in BMSi values between men and women in the group of acromegaly patients and in the control group. Although skeletal health has been shown to differ between the sexes, this observation is in keeping with other data on BMSi previously reported by our group (16) and another group (30), and partly in keeping with a study that investigated male and female HIV patients and controls and observed lower BMSi values in female HIV patients compared to male patients but comparable BMSi values between male and female controls (31).

In conclusion, we demonstrate that BMSi is significantly lower in patients with well-controlled acromegaly compared to controls. These data indicate that tissue-level properties of cortical bone remain significantly altered after cessation of long-term exposure to pathologically high GH and IGF-1 levels, probably contributing to the previously demonstrated ongoing increased risk for vertebral fractures. Future prospective studies addressing the skeletal complications of acromegaly should incorporate the study of microarchitecture and material properties of bone in addition to bone mass measurements.

\section{Supplementary data}

This is linked to the online version of the paper at http://dx.doi.org/10.1530/ EJE-16-0808.

\section{Declaration of interest}

The authors declare that there is no conflict of interest that could be perceived as prejudicing the impartiality of the research reported.

\section{Funding}

This research did not receive any specific grant from any funding agency in the public, commercial or not-for-profit sector.

\section{References}

1 Bonadonna S, Mazziotti G, Nuzzo M, Bianchi A, Fusco A, De Marinis L \& Giustina A. Increased prevalence of radiological spinal deformities in active acromegaly: a cross-sectional study in postmenopausal women. Journal of Bone and Mineral Research 200520 1837-1844. (doi:10.1359/JBMR.050603)

2 Wassenaar MJ, Biermasz NR, Hamdy NA, Zillikens MC, van Meurs JB, Rivadeneira F, Hofman A, Uitterlinden AG, Stokkel MP, Roelfsema F et al. High prevalence of vertebral fractures despite normal bone mineral density in patients with long-term controlled acromegaly. European Journal of Endocrinology 2011164 475-483. (doi:10.1530/EJE-10-1005)

3 Madeira M, Neto LV, Torres CH, de Mendonca LM, Gadelha MR \& de Farias ML. Vertebral fracture assessment in acromegaly. Journal of Clinical Densitometry 201316 238-243. (doi:10.1016/j. jocd.2012.06.002)

4 Claessen KM, Kroon HM, Pereira AM, Appelman-Dijkstra NM, Verstegen MJ, Kloppenburg M, Hamdy NA \& Biermasz NR. Progression of vertebral fractures despite long-term biochemical control of acromegaly: a prospective follow-up study. Journal of Clinical Endocrinology \& Metabolism 201398 4808-4815. (doi:10.1210/ jc.2013-2695)

5 Mazziotti G, Bianchi A, Porcelli T, Mormando M, Maffezzoni F, Cristiano A, Giampietro A, De Marinis L \& Giustina A. Vertebral fractures in patients with acromegaly: a 3-year prospective study. Journal of Clinical Endocrinology \& Metabolism 201398 3402-3410. (doi:10.1210/jc.2013-1460)

6 Roelfsema F, van der Sluys J \& Smeenk D. Quantitation of bone and bone turnover in biopsy specimens from the iliac crest in acromegaly. Journal of Endocrinology 197048 1xi.

7 Mazziotti G, Biagioli E, Maffezzoni F, Spinello M, Serra V, Maroldi R, Floriani I \& Giustina A. Bone turnover, bone mineral density, and fracture risk in acromegaly: a meta-analysis. Journal of Clinical Endocrinology \& Metabolism 2015100 384-394. (doi:10.1210/jc.20142937)

8 Bolanowski M, Daroszewski J, Medras M \& Zadrozna-Sliwka B. Bone mineral density and turnover in patients with acromegaly in relation to sex, disease activity, and gonadal function. Journal of Clinical Endocrinology \& Metabolism 200624 72-78. (doi:10.1007/s00774-0050649-9)

9 Madeira M, Neto LV, de Paula Paranhos Neto F, Barbosa Lima IC, Carvalho de Mendonca LM, Gadelha MR \& Fleiuss de Farias ML. Acromegaly has a negative influence on trabecular bone, but not on cortical bone, as assessed by high-resolution peripheral quantitative computed tomography. Journal of Clinical Endocrinology \& Metabolism 201398 1734-1741. (doi:10.1210/jc.2012-4073)

10 Mazziotti G, Bianchi A, Bonadonna S, Cimino V, Patelli I, Fusco A, Pontecorvi A, De Marinis L \& Giustina A. Prevalence of vertebral fractures in men with acromegaly. Journal of Clinical Endocrinology \& Metabolism 200893 4649-4655. (doi:10.1210/jc.2008-0791)

11 Maffezzoni F, Maddalo M, Frara S, Mezzone M, Zorza I, Baruffaldi F, Doglietto F, Mazziotti G, Maroldi R \& Giustina A. High-resolutioncone beam tomography analysis of bone microarchitecture in patients with acromegaly and radiological vertebral fractures. Endocrine 2016 54 532-542. (doi:10.1007/s12020-016-1078-3)

12 Ueland T, Ebbesen EN, Thomsen JS, Mosekilde L, Brixen K, Flyvbjerg A \& Bollerslev J. Decreased trabecular bone biomechanical competence apparent density, IGF-II and IGFBP-5 content in acromegaly, European Journal of Clinical Investigation 200232 122-128. (doi:10.1046/j.1365-2362.2002.00944.x)

13 Diez-Perez A, Guerri R, Nogues X, Caceres E, Pena MJ, Mellibovsky L, Randall C, Bridges D, Weaver JC, Proctor A et al. Microindentation for in vivo measurement of bone tissue mechanical properties in humans. Journal of Bone and Mineral Research 201025 1877-1885. (doi:10.1002/jbmr.73)

14 Guerri-Fernandez RC, Nogues X, Quesada Gomez JM, Torres Del PE, Puig L, Garcia-Giralt N, Yoskovitz G, Mellibovsky L, Hansma PK \& Diez-Perez A. Microindentation for in vivo measurement of bone tissue material properties in atypical femoral fracture patients and controls. Journal of Bone and Mineral Research 201328 162-168. (doi:10.1002/jbmr.1731) 
15 Farr JN, Drake MT, Amin S, Melton LJ III, McCready LK \& Khosla S. In vivo assessment of bone quality in postmenopausal women with type 2 diabetes. Journal of Bone and Mineral Research 201429 787-795. (doi:10.1002/jbmr.2106)

16 Malgo F, Hamdy NA, Papapoulos SE \& Appelman-Dijkstra NM. Bone material strength as measured by microindentation in vivo is decreased in patients with fragility fractures independently of bone mineral density. Journal of Clinical Endocrinology \& Metabolism 2015 100 2039-2045. (doi:10.1210/jc.2014-4346)

17 Biermasz NR, Pereira AM, Smit JW, Romijn JA \& Roelfsema F. Morbidity after long-term remission for acromegaly: persisting joint-related complaints cause reduced quality of life. Journal of Clinical Endocrinology \& Metabolism 200590 2731-2739. (doi:10.1210/jc.2004-2297)

18 Rikken B, van Doorn J, Ringeling A, Van den Brande JL, Massa G \& Wit JM. Plasma levels of insulin-like growth factor (IGF)-I, IGF-II and IGF-binding protein-3 in the evaluation of childhood growth hormone deficiency. Hormone Research 199850 166-176. (doi:10.1159/000023268)

19 Cole TJ. The LMS method for constructing normalized growth standards. European Journal of Clinical Nutrition 1990 44 45-60.

20 Genant HK, Wu CY, van KC \& Nevitt MC. Vertebral fracture assessment using a semiquantitative technique. Journal of Bone and Mineral Research 19938 1137-1148. (doi:10.1002/jbmr.5650080915)

21 Bridges D, Randall C \& Hansma PK. A new device for performing reference point indentation without a reference probe. Review of Scientific Instruments 201283 044301. (doi:10.1063/1.3693085)

22 Halse J, Melsen F \& Mosekilde L. Iliac crest bone mass and remodelling in acromegaly. Acta Endocrinologica 198197 18-22.

23 Lim SV, Marenzana M, Hopkinson M, List EO, Kopchick JJ, Pereira M, Javaheri B, Roux JP, Chavassieux P, Korbonits M et al. Excessive growth hormone expression in male GH transgenic mice adversely alters bone architecture and mechanical strength. Endocrinology 2015 156 1362-1371. (doi:10.1210/en.2014-1572)

24 Valassi E, Crespo I, Malouf J, Llauger J, Aulinas A, Marin AM, Biagetti B \& Webb SM. Reduction of trabecular and cortical volumetric bone mineral density at the proximal femur in patients with acromegaly. European Journal of Endocrinology $2016 \mathbf{1 7 4} 107-114$. (doi:10.1530/EJE-15-0931)

25 Duarte Sosa D \& Eriksen EF. Bone material properties are an independent determinant of fracture risk and vertebral fracture severity in osteoporosis. ECTS-IBMS Abstracts 2015 P29.

26 Rudang R, Zoulakis M, Sundh D, Brisby H, Diez-Perez A, Johansson L, Mellstrom D, Darelid A \& Lorentzon M. Bone material strength is associated with areal BMD but not with prevalent fractures in older women. Osteoporosis International 201627 1585-1592. (doi:10.1007/ s00198-015-3419-0)

27 Malgo F, Papapoulos SE, Hamdy NAT \& Appelman-Dijkstra NM. Bone Material Strength index in patients with low bone mass and fragility fractures. Osteoporosis International 201627 (Supplement 1) 48.

28 Duarte Sosa D, Vilaplana L, Guerri R, Nogues X, Wang-Fagerland M, Diez-Perez A \& Eriksen EF. Are the high hip fracture rates among Norwegian women explained by impaired bone material properties? Journal of Bone and Mineral Research 201510 1784-1789. (doi:10.1002/ jbmr.2537)

29 Godang K, Olarescu NC, Bollerslev J \& Heck A. Treatment of acromegaly increases BMD but reduces trabecular bone score - a longitudinal study. European Journal of Endocrinology $2016 \mathbf{1 7 5}$ 155-164. (doi:10.1530/EJE-16-0340)

30 Mellibovsky L, Prieto-Alhambra D, Mellibovsky F, Guerri-Fernandez R, Nogues X, Randall C, Hansma PK \& Diez-Perez A. Bone tissue properties measurement by Reference Point Indentation in glucocorticoid-induced osteoporosis. Journal of Bone and Mineral Research 201530 1651-1656. (doi:10.1002/jbmr.2497)

31 Guerri-Fernandez R, Molina D, Villar-Garcia J, Prieto-Alhambra D, Mellibovsky L, Nogues X, Gonzalez-Mena A, Guelar A, TrenchsRodriguez M, Herrera-Fernandez $S$ et al. Brief report: HIV infection is associated with worse bone material properties, independently of bone mineral density. Journal of Acquired Immune Deficiency Syndromes 201672 314-318. (doi:10.1097/QAI.0000000000000965)

Received 26 September 2016

Revised version received 20 December 2016

Accepted 10 January 2017 